\title{
Muscle Activity in Rat Locomotion: Movement Analysis and Electromyography of the Flexors and Extensors of the Elbow ${ }^{1}$
}

\author{
AVIS H. COHEN 2 AND CARL GANS \\ Department of Zoology, The University of Michigan, Ann Arbor, \\ Michigan 48104
}

\begin{abstract}
Footfall patterns and time sequence of activity are described for white rats conditioned to run freely in an activity wheel (which they drive). Motion is described in terms of soft contact, hard contact, soft contact, and flip phases. Duration of stride decreases and length of stride increases from walk to trot to canter to gallop. Myographic analysis shows that the brachialis has a major tonic function after it fires strongly during the flip phase and during much of the hard contact phase. Animals running at canter or gallop show major asymmetries between forelimb muscles on the first paw and on the lead paw sides.
\end{abstract}

Analysis of locomotion was, in the past, aimed primarily at large cursorial animals (Hildebrand, '62, '65; Howell, '44), but in recent years the situation has begun to change (Sukhanov, '68; Gambarian, '72). Taylor ('70) examined diverse forms of locomotion in the African viverrids, and Jenkins (71, '74) described the limb movements during walking in non-cursorial mammals, with an emphasis on primates and pointed out several cursorial specializations. Goslow and his colleagues studied hindlimb muscle lengths and joint angles in cats (Goslow et al., '73), correlating these with the electromyographic work of Engberg and Lundberg ('69). They also examined the nature and function of various hindlimb muscles (Goslow et al., '73; Cameron et al., '73).

To date, however, we lack a detailed analysis of the complete locomotion of a small terrestrial mammal. Domesticated rats and mice are used in enormous numbers of physiological and behavioral studies (see especially Barnett, '63; Sperry, '40, '41, '42; Zimmerman et al., '64), and changes in their locomotion are frequently monitored to determine the effect of particular procedures. Still, we have no good description of the locomotion patterns even of these ubiquitous laboratory animals, either in terms of footfall patterns or in terms of the muscular activities underlying these patterns, particularly in the forelimb.
A description of normal locomotion and of the muscular activities involved in it is of obvious interest. Such a baseline is absolutely essential for the characterization of differences between normal animals and those suffering from various muscle diseases or subject to experimental modification of muscle placement or innervation. It is also essential for discussion of the evolution of locomotor activities in terrestrial mammals. As a first step, we here attempt an analysis of the limb movements of laboratory rats running freely in an activity wheel. Following this, we will report on the activity of the flexor and extensor muscles that position and drive the elbow joint.

Our analysis proceeds from the general to the specific, outlining (1) general characteristics of the gaits, (2) the various footfall patterns observed, (3) the phases of the step cycle, and finally (4) the activities of the biceps, the brachialis, and the three heads of the triceps. Comparisons will be drawn between fast and slow gaits at each level of analysis.

\section{MATERIALS AND METHODS}

These experiments were performed on six male adult specimens of Rattus nor-

\footnotetext{
I Portions of this study were reported on at the 1974 meetings of the American Association of Anatomists in Cleveland, Ohio.

2 Present address: Department of Neurobiology and Behavior, Cornell University, Ithaca, New York 14853
} 
AVIS H. COHEN AND CARL GANS
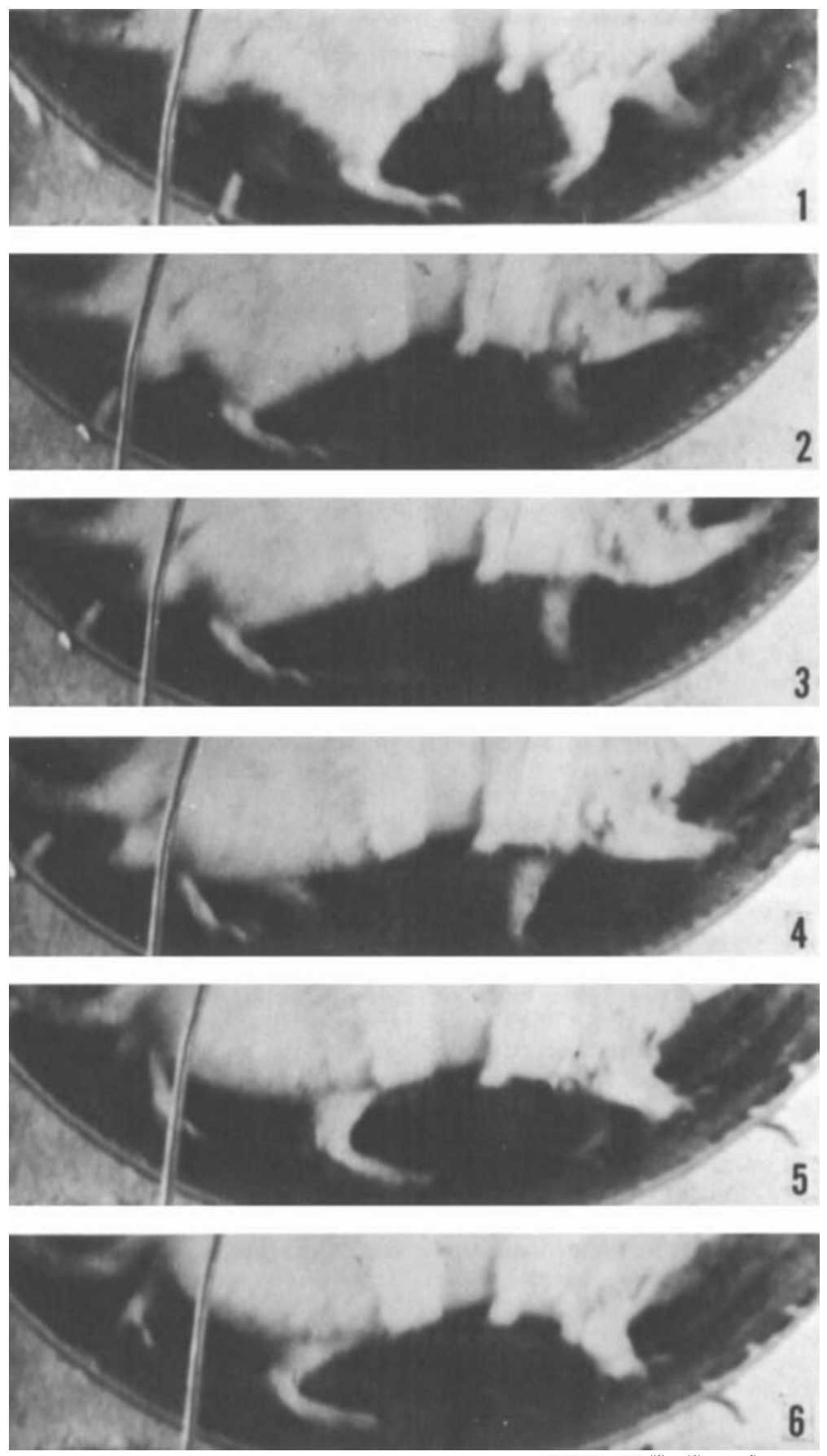

Figure 1A 

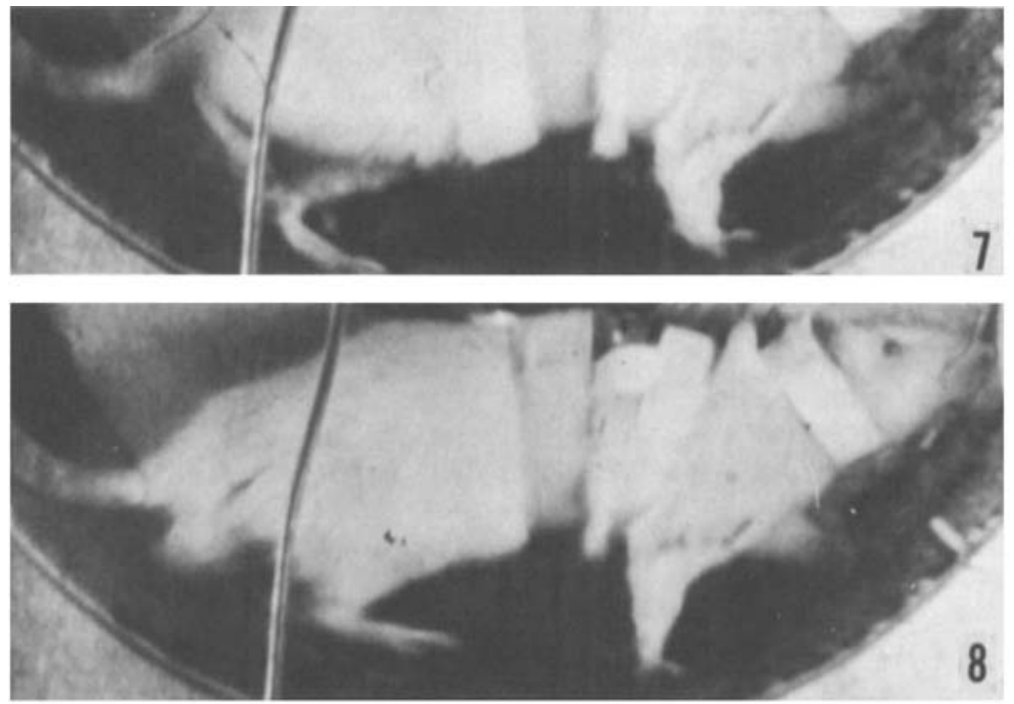

Figure 1A

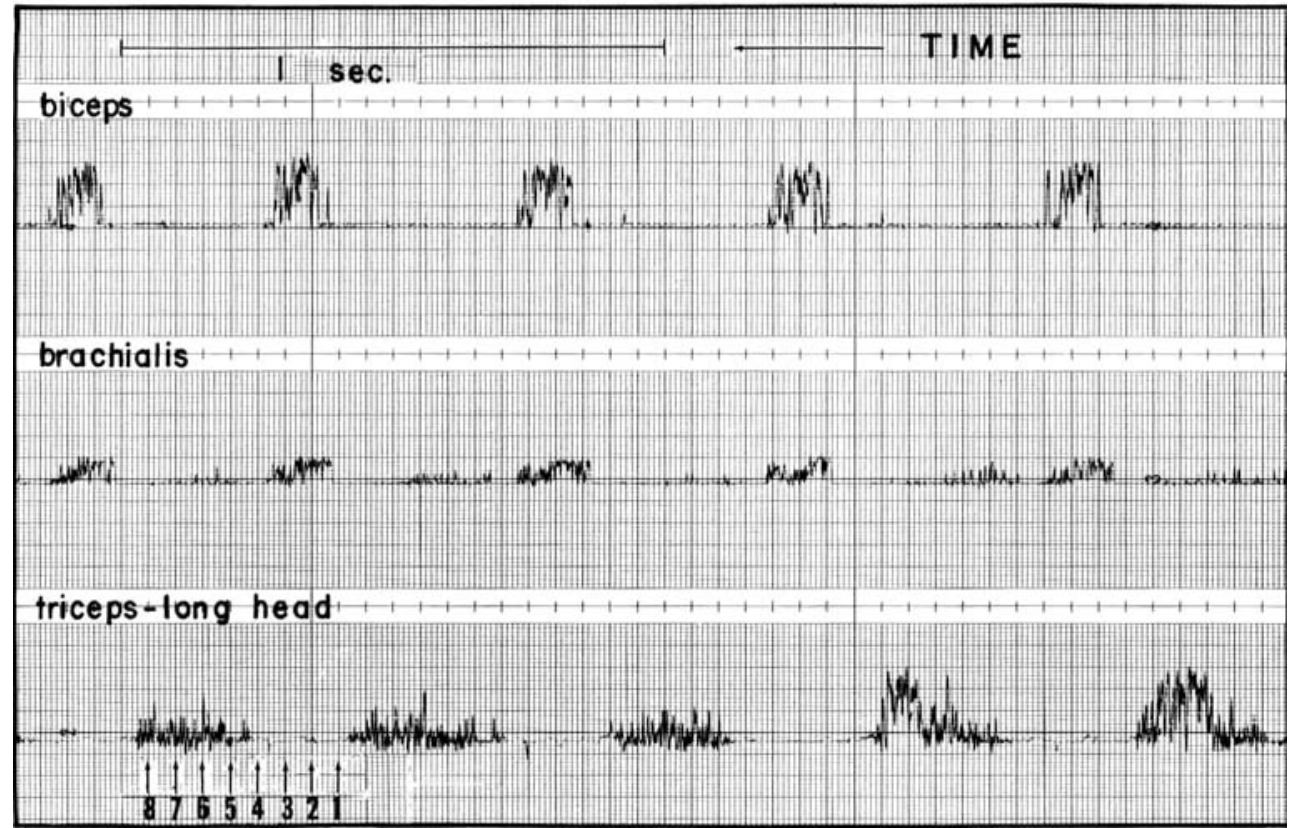

Figure $1 \mathrm{~B}$

Fig. 1 A single stride of a rat walking at approximately 2.5 strides per second. A. Prints (details of every third frame from record exposed at $64 \mathrm{fps}$ ) showing foot positions. B. Electromyogram for the stride sequence to show duration of activity in the biceps, brachialis and long head of the triceps muscles. The line indicates one second to scale and the vertical bars identify the instants when the frames shown in A were exposed.

vegicus (weighing 400 to 500 grams) taken from our colony of laboratory rats at The University of Michigan. The animals were conditioned to run unrestrained in an ac- tivity wheel (diameter $35 \mathrm{~cm}$; width $12 \mathrm{~cm}$ ) and were filmed from the side while moving. (As yet it has been impossible to utilize a wheel that would allow a simultane- 
ous frontal view.) After the animals had been conditioned to run freely, electrodes were implanted in the main muscles crossing the elbow and the leads drawn out through the back of the activity wheel. Pre- and post-implantation behavior were compared to avoid artifacts. Our general approach followed that of Gans ('74).

The animals were conditioned to run whenever the photoflood lights were turned on. Conditioning was by negative reinforcement provided by mild electrical shocks from a Grass PR 75 Stimulator, with the positive electrode connected to the animal and the negative to copper screening lining the activity wheel. This conditioned movement resulted in what appeared to be a natural motor pattern. The animals were allowed to drive the wheel (moving the wheel to force locomotion had produced apparently unnatural muscle activity in the untrained animal.)

Anesthesia was with veterinary Nembutal $(1 \mathrm{ml} / \mathrm{kg})$. The animals were shaved with a hair clipper. Electrodes of tefloncoated silver medwire $(0.003$ inch diameter) were inserted proximad into the muscle through a distally placed surgical incision. After placement the wires were kinked to provide stress relief in movement and threaded (via a large spinal needle) beneath the skin to a multi-pin connector that was taped to the skin at the nape of the neck. The local incision could then be sutured without stressing the deeply implanted wires or exposing the muscle to trauma. One prong of the multi-pin connector was attached to a ground wire placed in the loose subcutaneous tissue of the nape. The rats were permitted to recover for 10 to 12 hours, and were ordinarily tested for one hour. The electrodes had to be replaced for each experiment, because the animals' complex grooming activities often shifted the loose skin and dislodged or broke the underlying wires.

The filming and recording techniques were similar to those reported by Vree and Gans (75), except that the electronic strobe was used for film synchronization rather than illumination. Footfall patterns, step cycle, and muscular activity were analyzed, frame by frame, from 32 and 64 fps films of the rats (fig. 1). We analyzed only those sequences that showed at least eight strides. The elbow angle could not be de- termined, as the proximal portions of the upper limb apparently move beneath the loose skin of the trunk (cf. Jenkins, '71). Consequently the myograms were correlated with behaviorally distinguishable events in the step cycle (figs. 11, 12).

\section{RESULTS}

\section{Anatomy}

The general anatomy of the albino rat is well understood (Greene, '35). The following remarks are based on six freshly killed adult rats (400 to $500 \mathrm{~g}$ ) in which the overlying shoulder muscles had been removed and the elbow positioned at $70^{\circ}$, the approximate angle of the elbow in a standing animal (fig. 2).

The lateral head is the longest of the three heads of the triceps $(R=31-34 \mathrm{~mm}$, $\mathrm{m}=32.28$ ). It inserts along a $6-8 \mathrm{~mm}$ long segment of the olecranon process of the ulna; the other heads insert on the proximal end of this bone. The long head of the triceps is $29-32 \mathrm{~mm}(\mathrm{~m}=30.71 \mathrm{~mm})$ long and originates over a $9 \mathrm{~mm}$ segment of the scapula. The medial head originates from the ulna about $7.55 \mathrm{~mm}$ distal from the tubercle and is $22-26 \mathrm{~mm}$ long $(\mathrm{m}=24.57)$.

The brachialis is $24-29 \mathrm{~mm}(\mathrm{~m}=26.71$ $\mathrm{mm}$ ) long; it originates from the larger tubercle and the neck of the ulna and curves around the deltoid ridge to the medial side, inserting adjacent to the biceps tendon just distal to the coronoid process of the ulna. The major portion of the long head of the biceps is slightly longer $(R=$ $27-31 \mathrm{~mm}, m=29.43$ ); it arises from the anterior edge of the glenoid cavity and inserts on to the tuberosity of the radius.

\section{Movement analysis \\ General characteristics of the gaits}

In an activity wheel, rat gaits fall into two clearly distinguishable categories, best characterized by a difference in speed and symmetry of footfalls; a further separation by speed into two subcategories is convenient for analysis but implies no further qualitative distinction. Thus the walk and trot are slower and more often symmetrical, while the canter and gallop are faster and more often asymmetrical. It must be emphasized that although these terms are useful for descriptive purposes, they oversimplify the actual conditions observed even in an activity wheel. These gaits pre- 


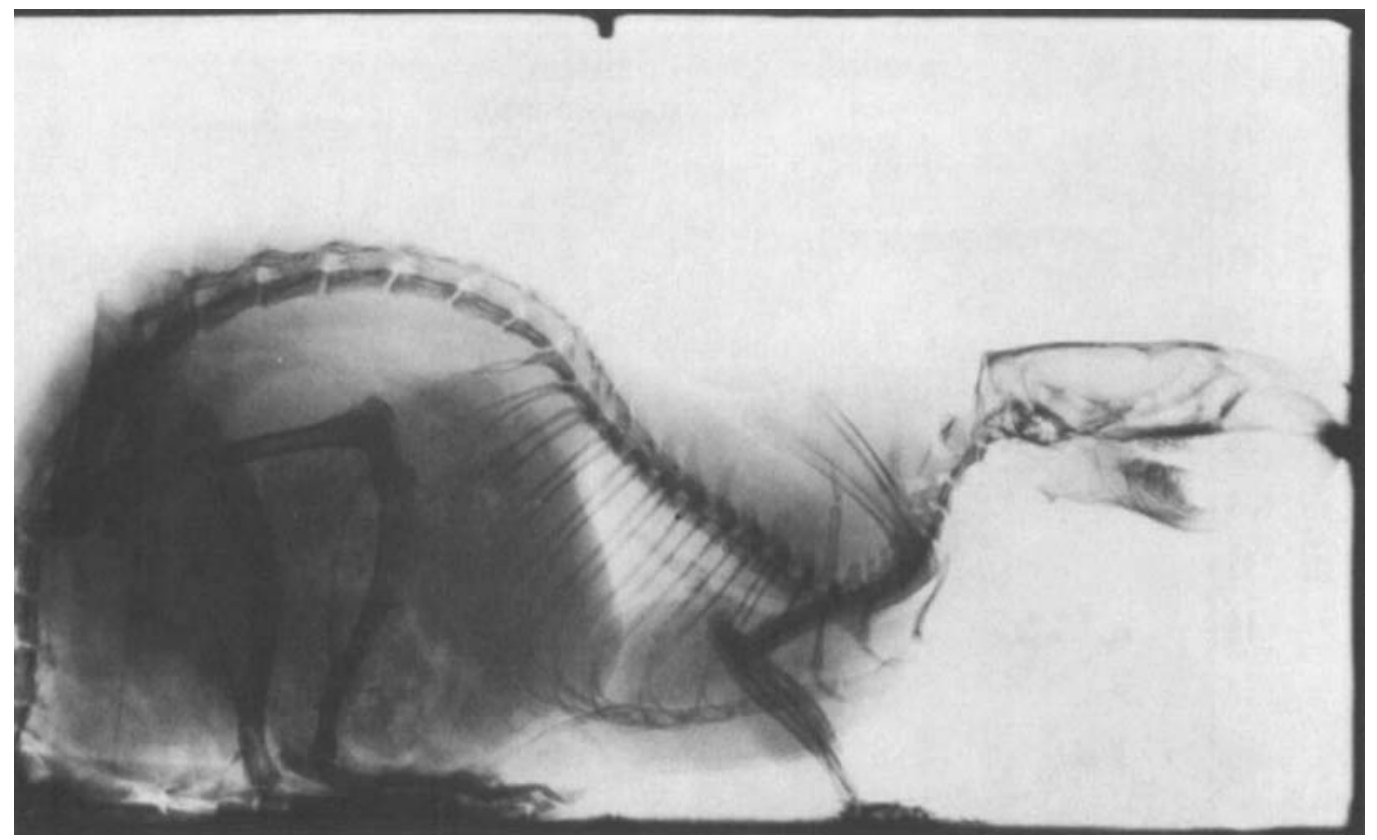

Fig. 2 Lateral radiograph of a standing rat. Note the angle of the elbow.

sumably represent only a small portion of the animals' normal repertoire under natural conditions (Ewer, 71). One does note general trends across the categories; these trends illustrate the rats' locomotor style and the adaptations of these animals for achieving increased speed.

The most common gait appears to be some form of trot; only one of our animals consistently galloped (ran with an asymmetrical gait at the highest speeds observed). The canter is more common than the gallop; it shows many of the characteristics of the gallop and differs mainly in their expression.

The speed per stride ranges from a low of $31.9 \mathrm{~cm} / \mathrm{sec}$ for a slow walk to a high of $125.9 \mathrm{~cm} / \mathrm{sec}$ for a gallop. The galloping animal never walked at less than $43.0 \mathrm{~cm} /$ sec. (These figures are all taken from a sequence of at least eight consecutive strides, and represent neither standing nor lurching.) The time required for a stride ranges from a high of $500 \mathrm{msec}$ for a walking animal to a low of $190 \mathrm{msec}$ for the galloping one. The distance covered in a stride ranges from a minimum of 11.1 $\mathrm{cm}$ during the walk to a maximum of 34.5 $\mathrm{cm}$ during the gallop. Both time and distance per stride vary a great deal within a given gait. In one animal, a clearly recognizable trot produced speeds between $37.5 \mathrm{~cm} / \mathrm{sec}$ and $87.9 \mathrm{~cm} / \mathrm{sec}$. Since confinement in laboratory cages does not permit much exercise, the maximum speeds reported here are almost certainly less than those achieved by animals that are able to exercise regularly.

When the full range of gaits is plotted for one animal (figs. 3, 4), one notes an increase in length (and rate) of stride and a decrease in duration of stride; these result in an increase in the absolute velocity of the animal. A shift from walk to gallop increases the stride length by $47 \%$ and decreases the stride duration $41 \%$, to increase the speed by $142 \%$.

This result is contrary to the previously held belief that small animals increase stride length while decreasing stride rate (i.e., increase stride duration) to gain speed (Hildebrand, '62). In addition, the rats achieve additional speed by back flexion and extension, thus lengthening the stride. In a single isolated bound the increased stride rate may perhaps not be apparent, but this trend holds constant across all animals and all gaits observed. Increased speed, then, results from an increase of stride length and of stride rate. 


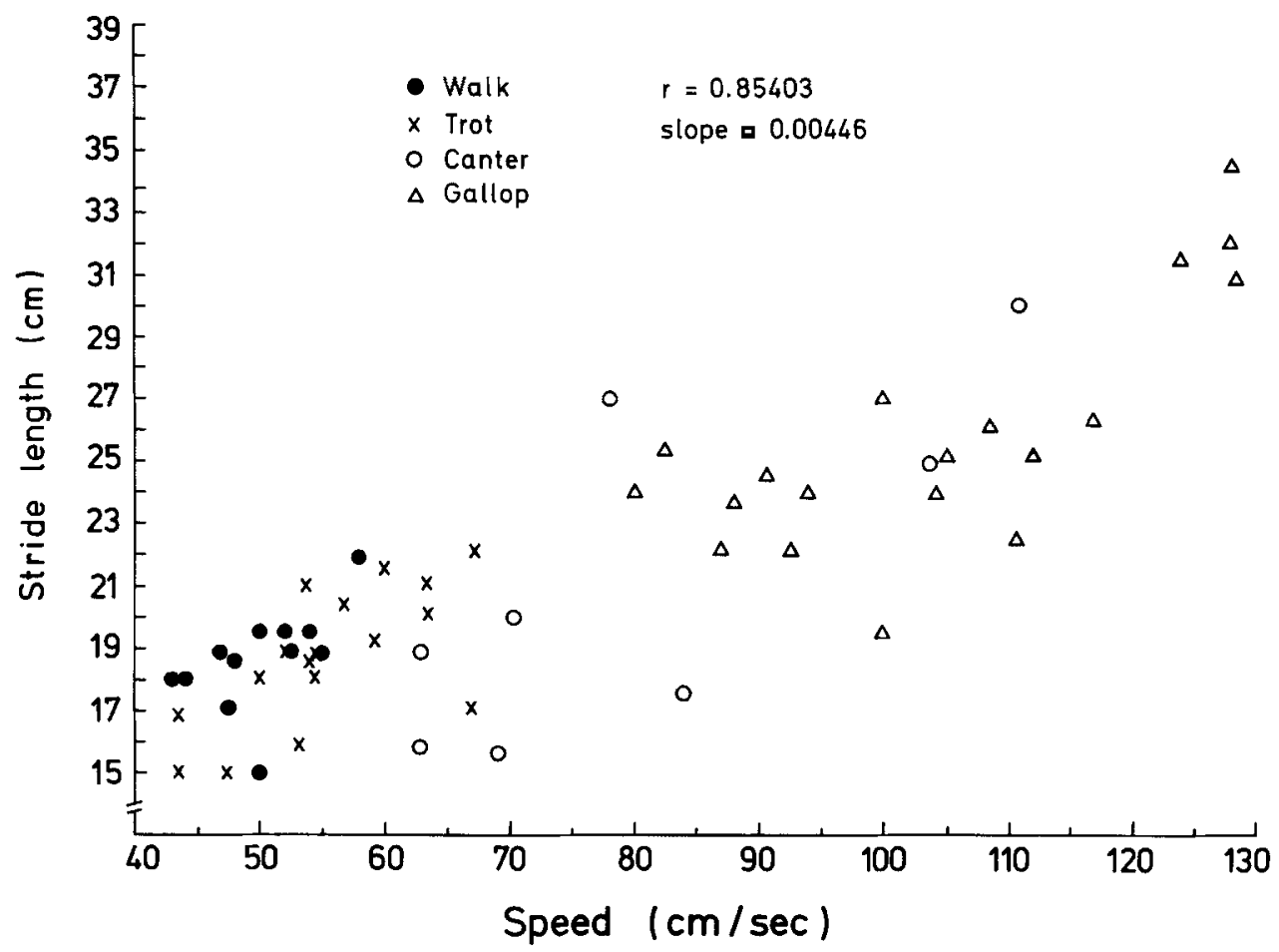

Fig. 3 Length of stride plotted against speed. Data taken from one rat.

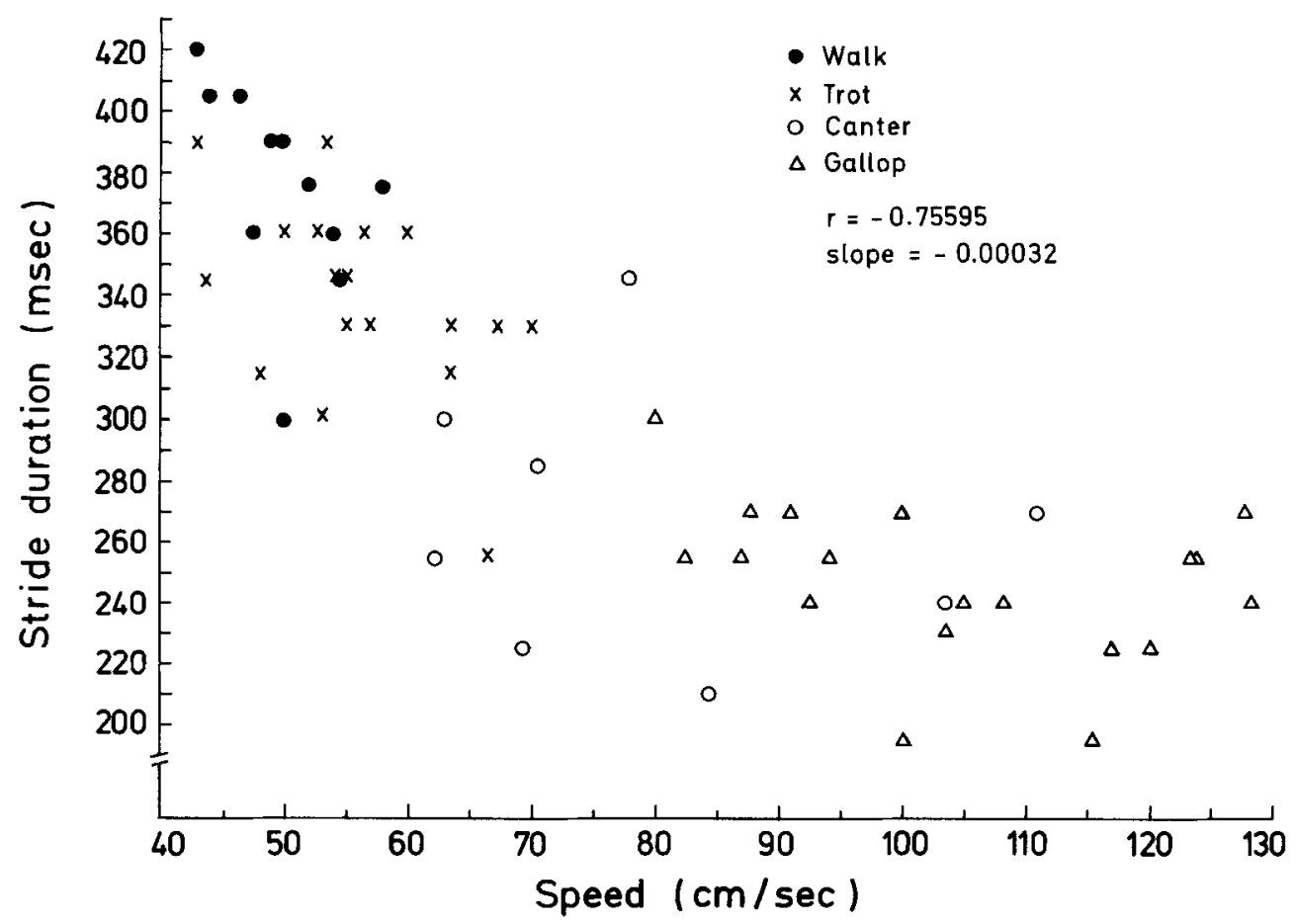

Fig. 4 Duration of stride plotted against speed. Data from the same rat as in figure 3 . Stride rate is the inverse of stride duration; therefore, speed is positively correlated with stride rate. 


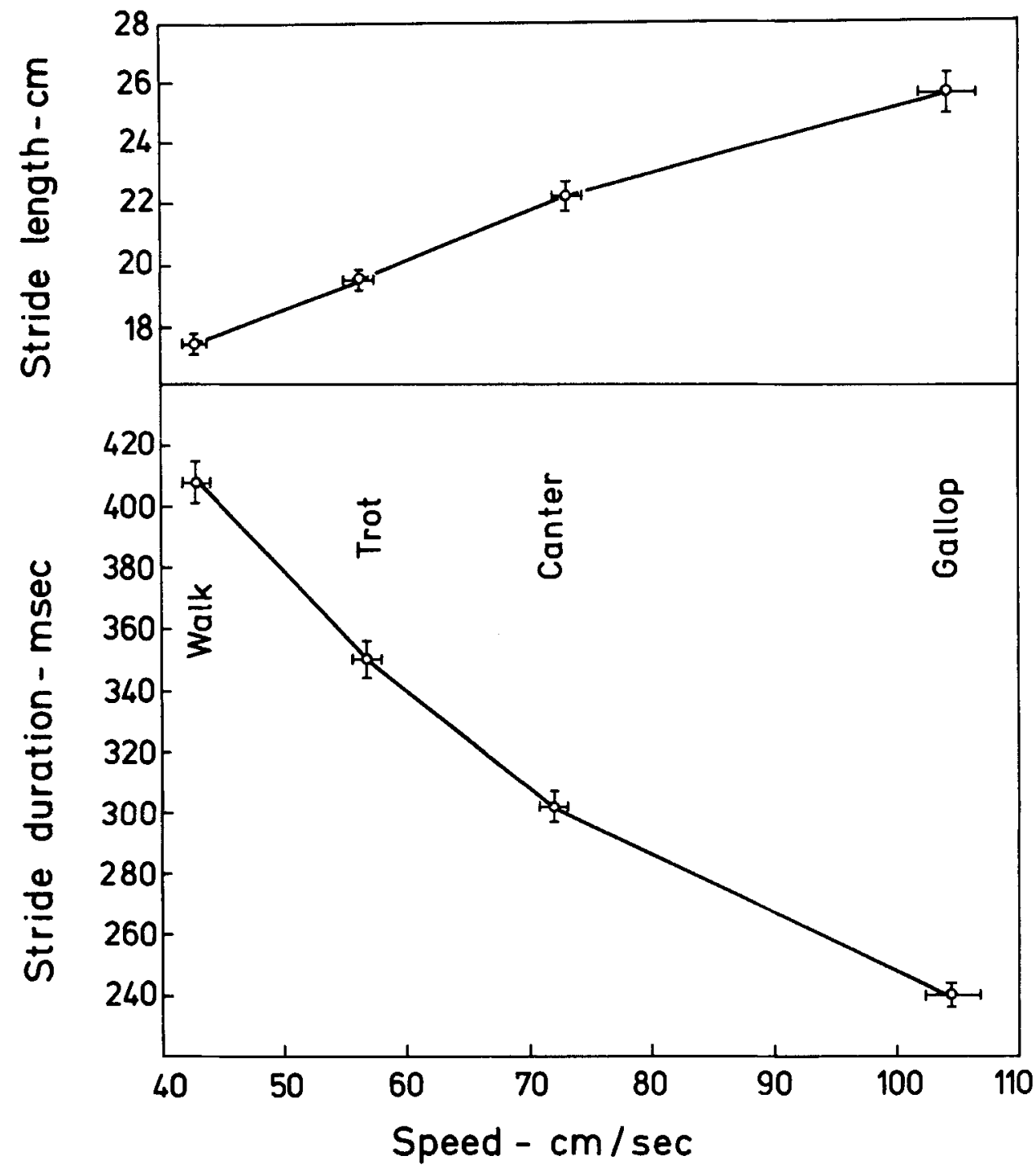

Fig. 5 Mean length of stride (a, top) and mean stride duration (b, bottom) plotted against mean speed for all animals. Comparison shows that stride duration is negatively correlated with stride length.

Stride length is relatively more important at the faster gaits in which the animal adopts a rapid bounding movement, utilizing the stronger hindlimbs to a greater extent.

\section{Footfall patterns}

Hildebrand's ('66) terminology for symmetrical gaits is the most convenient to describe the slower gaits; it is used here.

The slower gaits are the most variable, but all animals use some form of lateral sequence gait. Most use a single foot or diagonal couplet walk or trot. One animal more often uses a lateral couplet walk with frequent transitions to a single-foot gait. Examples of the characteristic slower gaits of three animals are presented in figure 6 to illustrate the range of footfall patterns. The animals always have two and often three feet on the ground; the forefoot lands subsequent to the ipsilateral hind foot. The percentage of stride time between hind foot and ipsilateral forefoot ranges from 

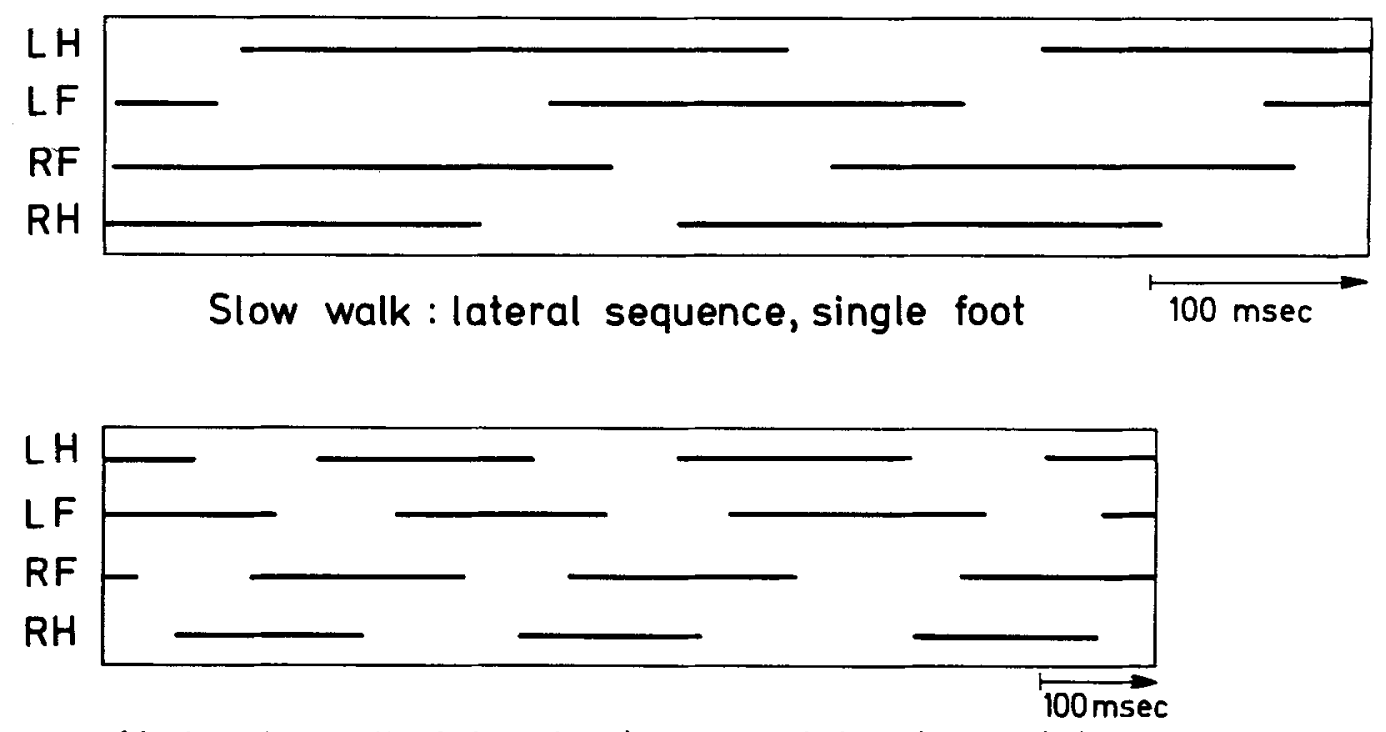

Moderate walk: lateral séquence, lateral couplet

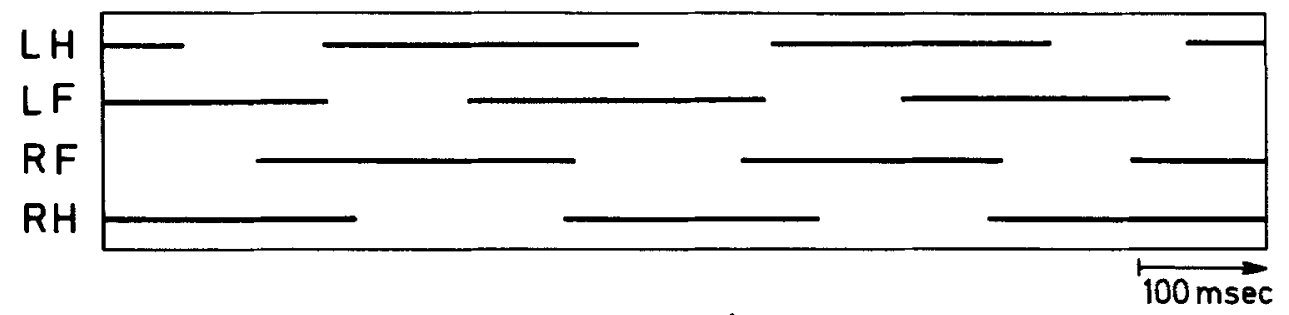

\section{Fast walk: lateral sequence, diagonal couplet}

Fig. 6 Three of the symmetrical footfall patterns seen in rats. Each example is from a different animal. $L, R, H$, and $F$ represent left, right, hind and fore foot. Line marks the time during the stride that the foot is on the ground. Time goes from left to right, with the actual time indicated by a time marker for each.

$14 \%$ (lateral couplet) to $50 \%$ (diagonal couplet). The hind foot is in contact with the ground during $50 \%$ of the stride in a fast walk, and during $75 \%$ in a slow walk.

As the speed of an animal increases, the percentage of time that the hind foot contacts the ground decreases. The footfall pattern simultaneously becomes increasingly asymmetrical, and one can discern a lead paw, the paw which is clearly the second of the (anterior or posterior) pair to leave the ground. At its fastest, the gait becomes a transverse gallop, with the front and rear lead foot being on the same side. The hind paws contact the ground nearly simultaneously; the forepaws strike nearly together in a bound; less often, they strike more alternately in a half-bound. Occa- sionally, all four paws are off the ground simultaneously, although the more common pattern is no more than three at once. Figures 7 and 8 give two examples of short sequences illustrating the bound and halfbound.

The canter is often a transitional gait. It frequently occurs when an animal begins to speed up while trotting until it "breaks stride," to borrow from the horse terminology; that is, the animal's gait becomes asymmetrical. For those animals that rarely gallop, the canter occurs intermittently during fast trot sequences. The galloper uses it both with the trot and as a way of speeding up to a full gallop. An example of the canter is given in figure 7 . Because of its transitional character, this 

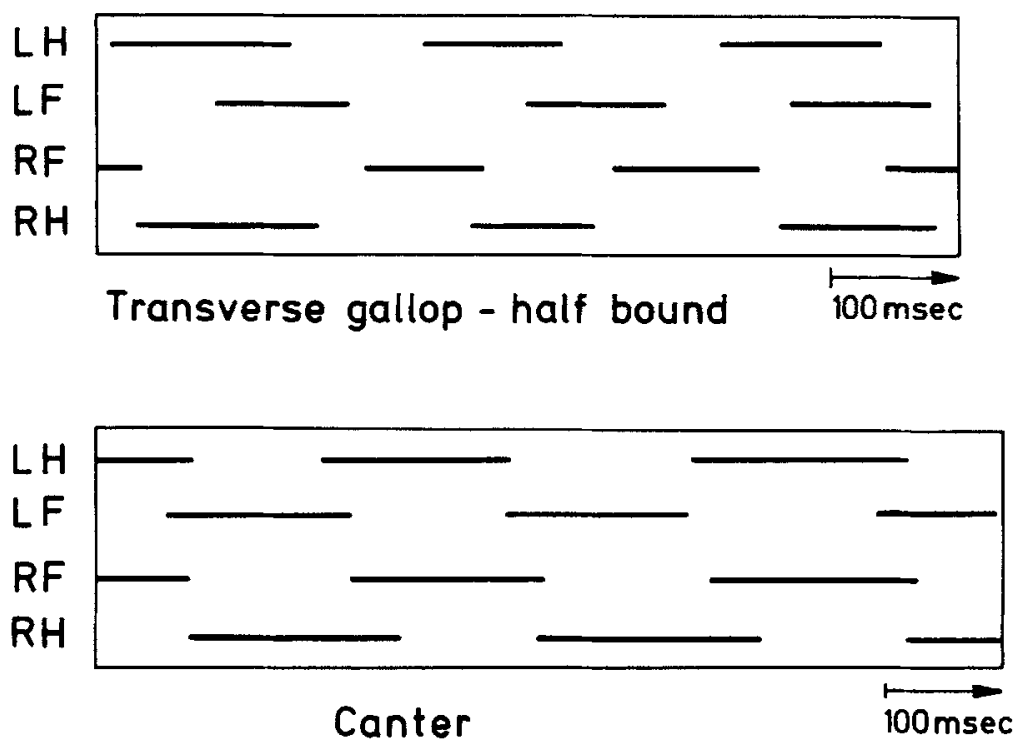

Fig. 7 Examples of asymmetrical footfall patterns of two rats. Conventions as in figure 6.
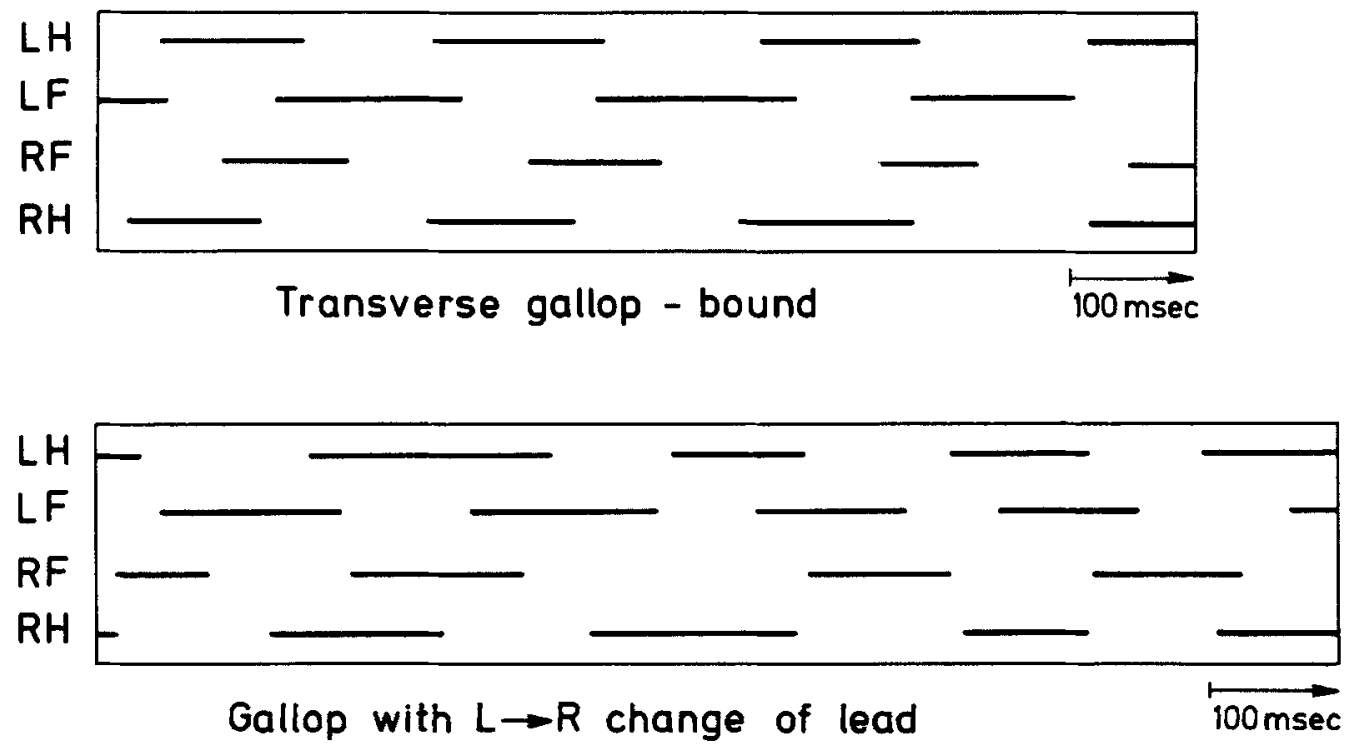

Fig. 8 Examples of asymmetrical footfall patterns from one rat; (b) lower figure illustrates a change of lead in the middle of the sequence.

gait is hardest to delineate clearly. For analysis, we only use sequences of strides that are asymmetrical and generally faster than the trot but are not yet a full gallop. The greatest qualitative differences are between symmetrical and asymmetrical gaits. Thus, the somewhat arbitrary sepa- ration into canter and gallop is mainly a convenience for discussion.

Some animals appear to have a preferred lead; for others the data are inconclusive. Some rats change leads (fig. 8). In the example shown, the lead change is accomplished by the left forelimb rapidly passing 
the right, while the hindlimbs pick up the new lead; this gives the left hindlimb a shortened period on the ground.

\section{Phases of the step cycle}

The step cycle represents the sequence through which each limb passes as it is alternately in contact with the ground and lifted out of contact. The step cycle is most usefully divided into four phases, distinguishable on the basis of behavioral criteria that correspond to functionally important transitions in the cycle. (1) Soft contact -1 is the period following the forward and downward movement of the paw. It begins with initial ground contact and terminates with subsequent full ground contact. Towards the end of this phase the propulsive function of the limb begins. (The start of vertical and horizontal force transmission remains to be determined, perhaps by a force plate analysis.) (2) Hard contact (more commonly stance) is the phase when the paw has maximum surface contact and is weight bearing. (3) Soft contact-2 marks the beginning of the forward movement of the paw. Contact is maintained, but propulsion ceases during this phase. The wrist begins to dorsiflex and the limb lifts at the end. (4) The flip (or swing) phase starts when the manus leaves the ground, and is flexed to avoid dragging. The elbow is also flexed and the limb moves forward both absolutely and relative to the animal. At the end of this phase the manus is extended and dropped. During the faster gait the flip phase includes a very active reaching prior to placement. The combined limb movements are more active that "swing" would imply. These four phases correspond roughly to those described by Jenkins ('71) in his study of non-cursorial mammalian locomotion.

Each step cycle may be subdivided by noting three clearly distinguishable events. Other points are more difficult to measure reliably. These clear events are (1) the initial paw contact $\left(S_{1}\right),(2)$ the instant when the limb starts to move forward and the wrist to dorsiflex, distinguished behaviorally by the lifting of the back of the pad out of contact and a simultaneous anteriad shift of the limb $\left(\mathrm{S}_{2}\right)$, and (3) the lifting of

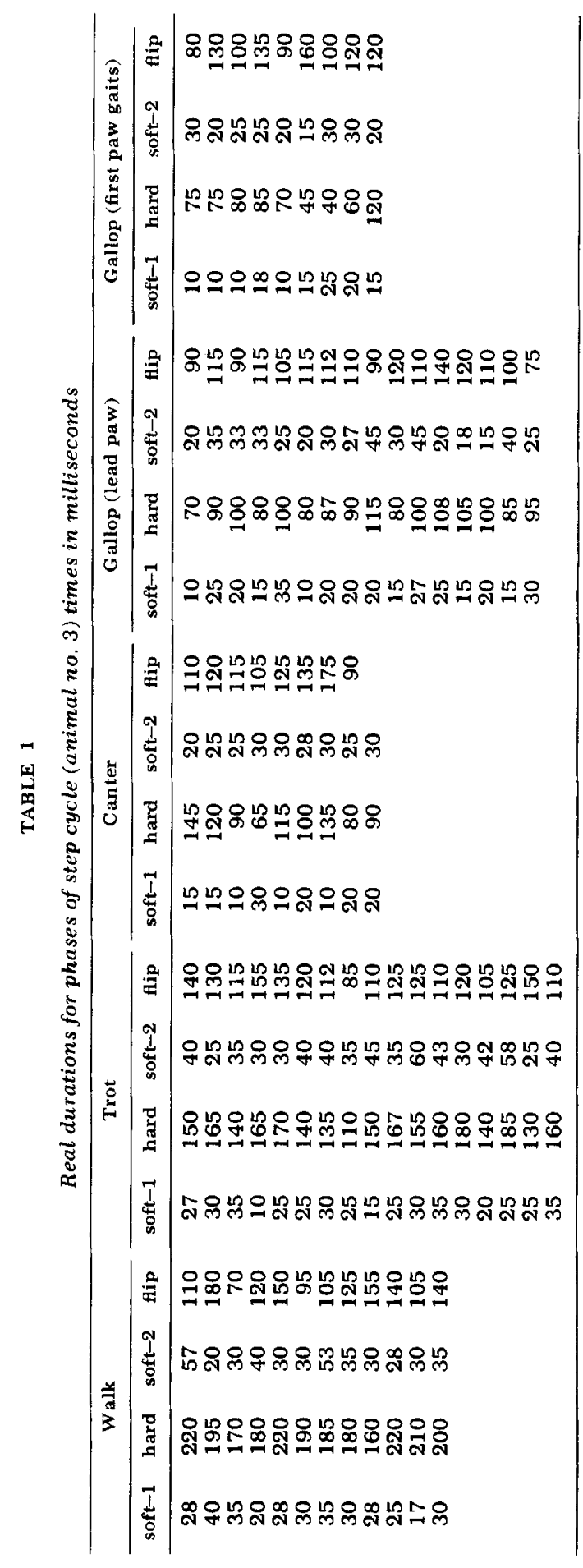




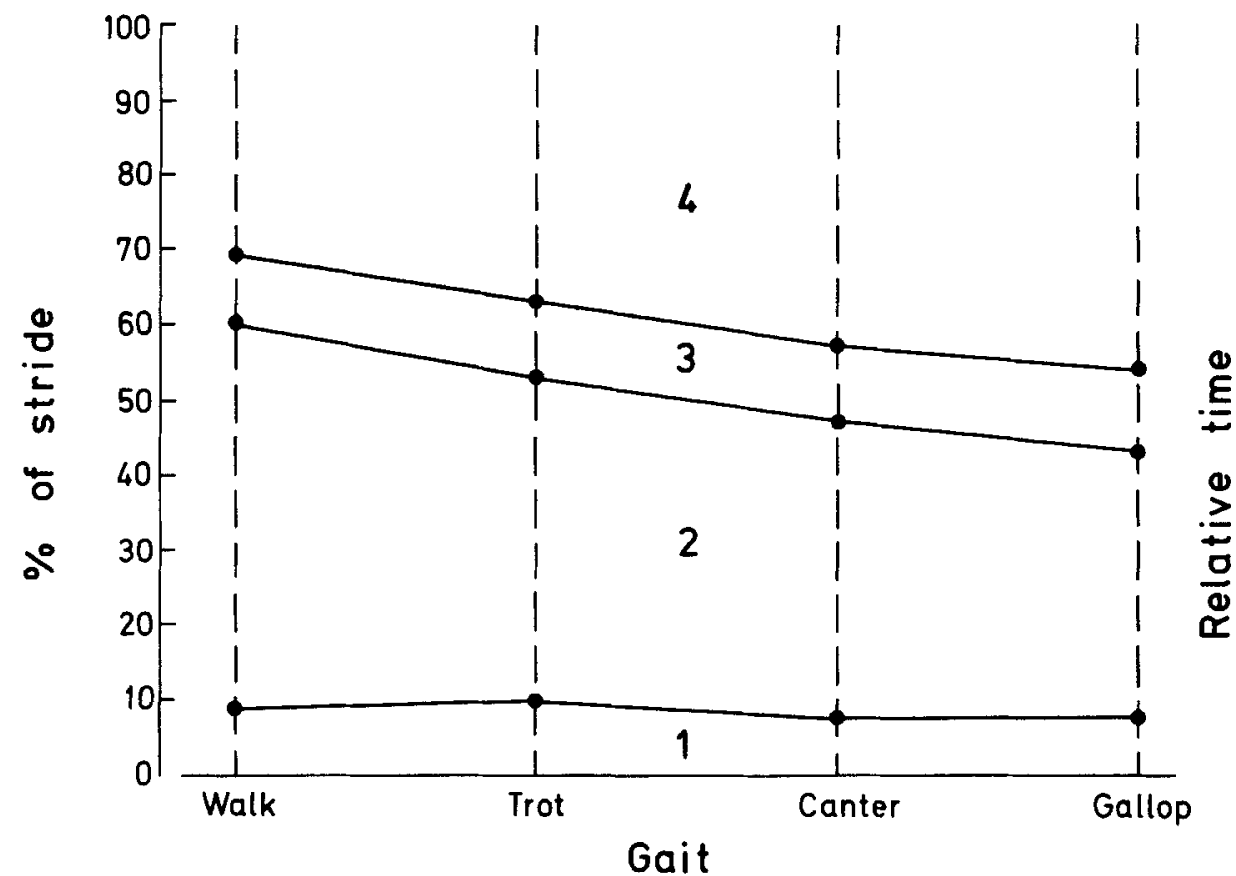

Fig. 9 Changes in the relative duration for the phases of the step cycle of rats across gaits. Vertical lines represent $100 \%$ of the step cycle for the indicated gait. Points are placed at relative time in the cycle that phase begins. Thus in the walk, phase 1 ends at $9 \%$, phase 2 ends at $60 \%$, assuming $51 \%$ of the cycle, phase 3 ends at $70 \%$ and phase 4 fills the remainder, assuming $30 \%$ of the cycle. Solid lines connect the beginning points of the same phase across gaits. Phase 2 (hard-contact) drops from $51.7 \%$ of the cycle during the walk to $35.2 \%$ in the gallop, with a corresponding increase in phase 4 (flip phase). Changes in phases 1 and 3 are within observational error.

the paw out of contact with the ground (off).

The actual time of each of the phases is extremely variable, because of the variability in stride duration (fig. 4). However, the variability decreases and comparisons between gaits become feasible when the phases are expressed in percent of each stride's duration. The phase times of the one rat that used all four gaits are listed in table 1 to permit an estimate of the actual magnitudes. Figure 9 illustrates the mean percentage of stride interval for each phase of the four gaits (data from the same animals as figure 5). Soft contact -1 takes about $9 \%$ of the stride during the slower gaits and about $7.5 \%$ during the faster gaits. This difference, which amounts to about 3 to $5 \mathrm{msec}$, is well within observational error. Soft contact -2 similarly shows only a small ( $3 \%$ of stride interval) change from walk to gallop; this too can be attributed to error. With increased speed, the time occupied by the hard contact phase steadily declines from $51.7 \%$ in the walk to $43.5 \%$ in the trot. For the canter, hard contact shows a mean of $38.4 \%$; for the gallop, $35.2 \%$. This large drop reflects an increase in the relative length of the flip phase, which increases from $30.9 \%$ for the walk, to $36.7 \%$ for the trot, $43.5 \%$ for the canter, and $45.5 \%$ for the gallop. This $15 \%$ overall increase clearly correlates with the increased stride length at the faster gaits. The additional time during the flip phase is occupied by the reaching out of the paw prior to placement. When the data for the gallop are analysed separately for the lead and first paw (fig. 10), the first paw shows a greater relative increase in the duration of the flip phase than did the lead paw. This suggests that the first paw to strike the ground establishes an upper limit on stride length even 


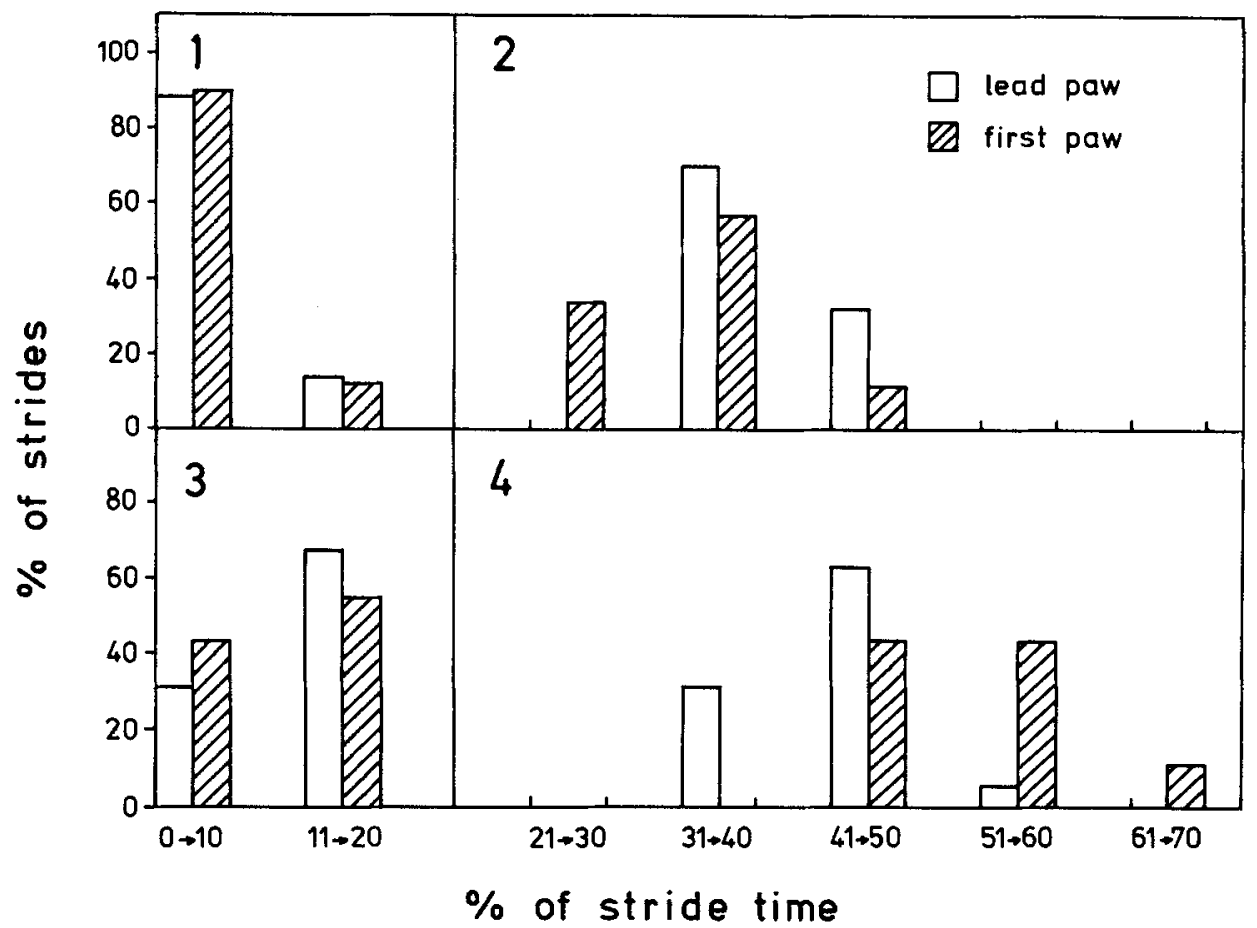

Fig. 10 Differences in the phases between "first" and "lead" paw in asymmetrical gaits. The abscissa is divided into 10 percentile partitions as indicated. The data are the same as figure 8 for canter and gallop, separating the strides on the basis of "first" and "lead" paw.

when the animal is progressing by half bounds with the hindlimbs providing a great deal of the forward thrust.

\section{Muscle activity}

\section{General}

The onset and cessation of muscular activity were mapped versus the three sharply distinguishable points, $S_{1}, S_{2}$, and "off" of particular step cycles. Differences in levels of firing are only reported when they consistently occurred in multiple preparations. Several muscles occasionally and irregularly fired at other times; the more variable activities will not be dealt with at this time.

\section{Flexors}

The biceps and brachialis both commence firing during soft contact -2 . In the slow gaits, activity in the brachialis precedes that in the biceps and generally starts $10-19$ msec after $S_{2}$ (fig. 11) regardless of the duration of the stride. The onset of activity in the biceps correlates poor- ly with this marker; however it usually follows the activity in the brachialis. It shows a better distribution about "off," the break in ground contact (fig. 11), being centered at 11-20 msec before this marker. In the fast gaits, the brachialis fires nearer "off," while activity of the bi-

Fig. 11 Activity of the flexors biceps long head and brachialis in the rat. The abscissa of each histogram in time relative to the event in the step cycle indicated by the vertical bar above the 0-9 segment. Positive numbers represent time after the respective event, negative before. $S_{2}$ is the beginning of soft contact -2 phase, $S_{1}$ is initial ground contact, i.e., the beginning of soft contact - 1. Off is break in ground contact. The ordinate is number of strides, or number of times the muscle began or ceased firing relative to event indicated. The two histograms of flexor onset utilize the same strides, with only the marker changed. Note the altered distribution of the biceps relative to $S_{2}$ and off, and the comparative lack of change from fast to slow gaits (although this implies a shift in the percentage of the cycle when they are active). These data are only for high levels of activity and do not include low-level brachialis activity or the less consistent low-level biceps activity that is seen during the hard-contact phase. 

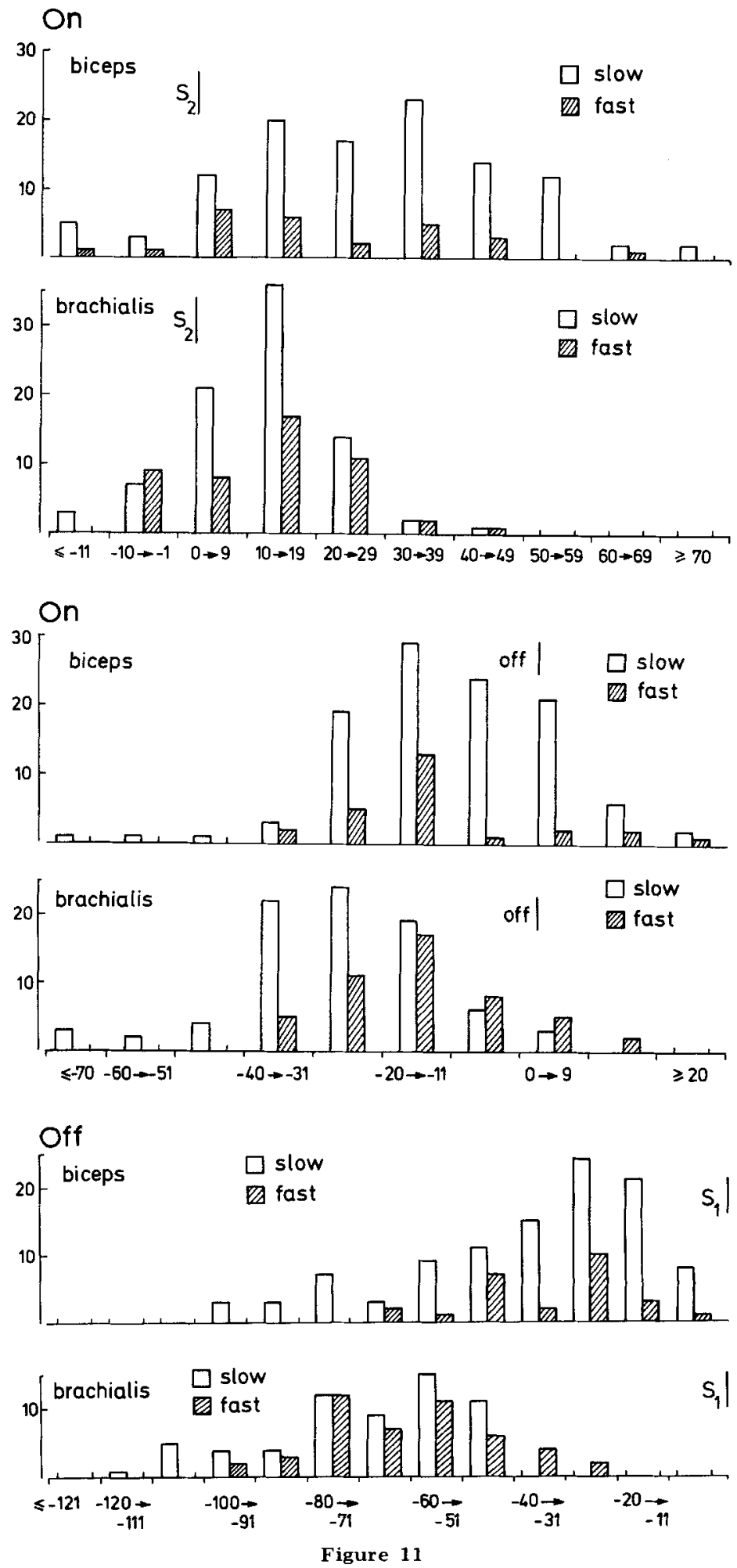


\section{Triceps - off}
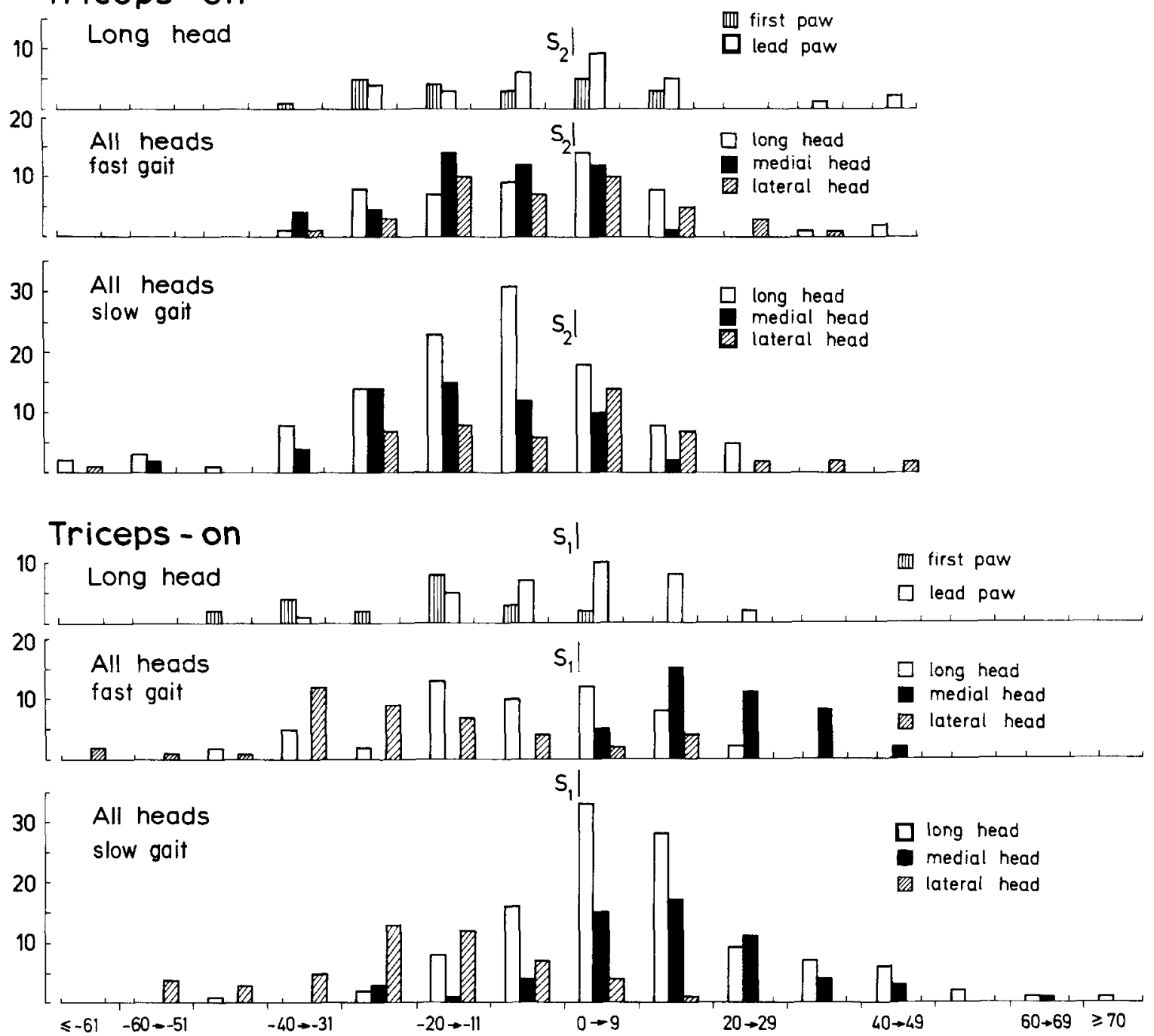

Fig. 12 Activity of the extensors triceps long head, medial head, and lateral head. Conventions as in figure 11. All three heads are included where indicated. Data from the long head are separated into "first" and "lead" paw categories, since these showed clear asymmetry.

ceps remains unchanged. This means that they fire essentially simultaneously during the asymmetric gaits.

$\mathrm{S}_{1}$ follows the end of high-level firing in the brachialis by some $60-70 \mathrm{msec}$; the biceps ceases firing $30 \mathrm{msec}$ before this (fig. 11). These relationships apparently do not change when the animal shifts to the faster gaits, even though the flip phase is proportionally longer in the canter and gallop. This means that these muscles must fire for a greater percentage of the phase.

When the rat progresses slowly, the brachialis continues to fire at a low level until about $20 \mathrm{msec}$ before $\mathrm{S}_{1}$; as the rat shifts to a faster gait, the muscle fires until $S_{1}$. This muscle also fires at a low level during hard contact. The duration of firing varies within this phase when the rat moves slowly; the onset of low level activity precedes hard contact during fast gaits. 
In a few strides the brachialis never ceased firing in the transitions from flip phase to soft contact -1 to hard contact.

\section{Extensors}

In the walk and trot, the long head of the triceps starts to fire less than $9 \mathrm{msec}$ after $S_{1}$ and continues its activity until 10 msec or less before $S_{2}$. The medial head follows at 10-19 msec after $S_{1}$ (fig. 12) and stops firing about $20 \mathrm{msec}$ before $S_{2}$. The lateral head has two types of actions: it fires at a low level beginning 20-30 $\mathrm{msec}$ before $\mathrm{S}_{1}$; some $30 \mathrm{msec}$ before cessation of firing by the medial head (just before $S_{1}$ ), the lateral head sharply increases its activity for $30 \mathrm{msec}$, and then fires at low level for another 10-20 msec.

During canter and gallop the medial head maintains its temporal relationships to $S_{1}$ and $S_{2}$. As the rat speeds up, the lateral head shifts its onset to $40 \mathrm{msec}$ prior to $S_{1}$. The data do not permit clear statements whether this muscle fires at the same time in the lead paw as in the first paw. However, the long head shows a different firing sequence in the lead and in the first paw; these activities are almost completely discrete (fig. 12). The long head of the first paw tends to start firing 20 msec before $S_{1}$, whereas the long head of the lead paw fires at $S_{1}$. The long head of the lead paw also fires longer than that of the first paw (fig. 12).

Figures 13 and 14 illustrate these temporal relations in a composite polar diagram; the step cycle is represented as a circle, with the phases marked according to their estimated percentages of the stride for generalized slow and fast gaits.

\section{DISCUSSION}

\section{Functional morphology}

The mammalian forelimb functions during locomotion to steer the animal and to keep the anterior body and head off the ground. It also acts as a propulsive agent, maintaining velocity or contributing to acceleration. It must be recognized that moving on a treadmill or a small radius activity wheel represents one of the simplest aspects of a complex and highly adaptable grouping of motor sequences. The animal runs at different velocities, but it does not have to compensate for un- eveness of the ground, nor for sudden obstacles. Our system does not consider the effects of uneven ground texture in terms of support or traction, nor does it deal with change of direction. (The wheel's inertia adds another complication.)

Movement analysis permits us to make some first level quantitative statements about the functional subdivision of the step cycle; it suggests differentiation between the supportive and the propulsive portions and the separation of both of these from that portion of the stride when the foot merely touches the ground, transmitting only insignificant forces. We have documented this by subdividing the contact time into soft-hard-soft; the reality of this subdivision is supported by the changes in muscular activity correlated with these transition points.

In the following discussion we are obviously simplifying certain relations, the support-propulsion relations in particular. As long as the chain of links comprising a limb is stabilized (kept from lateral and anterposterior buckling) at the joints, each pair of links will be able to transmit compressive forces across the encapsuled articulation. Such forces are borne by the bones rather than transmitted through the contracting muscles. Consequently the muscles may serve a major function in positioning the elements; however, the magnitude of their activity does not necessarily reflect the magnitude of supportive forces. The anterior limb only serves propulsion when it is retracted faster than the trunk is advanced (due to the momentum imparted by the hindlimbs). The following speculations hence remain to be tested by direct recording of the vertical and horizontal forces transmitted by the limbs.

The rotation of the limb through a step cycle may be divided into several phases. The movements and forces characterizing each phase can as yet be only incompletely correlated since we are considering only the muscles bridging the elbow. For instance, the firing level of the protractors shows unexplained variation during the middle of the flip phase; this may reflect positional and other intercycle differences not characterized by this simple scheme.

The extensor muscles fire at or just before the beginning of the hard contact phase and cease activity before its termina- 


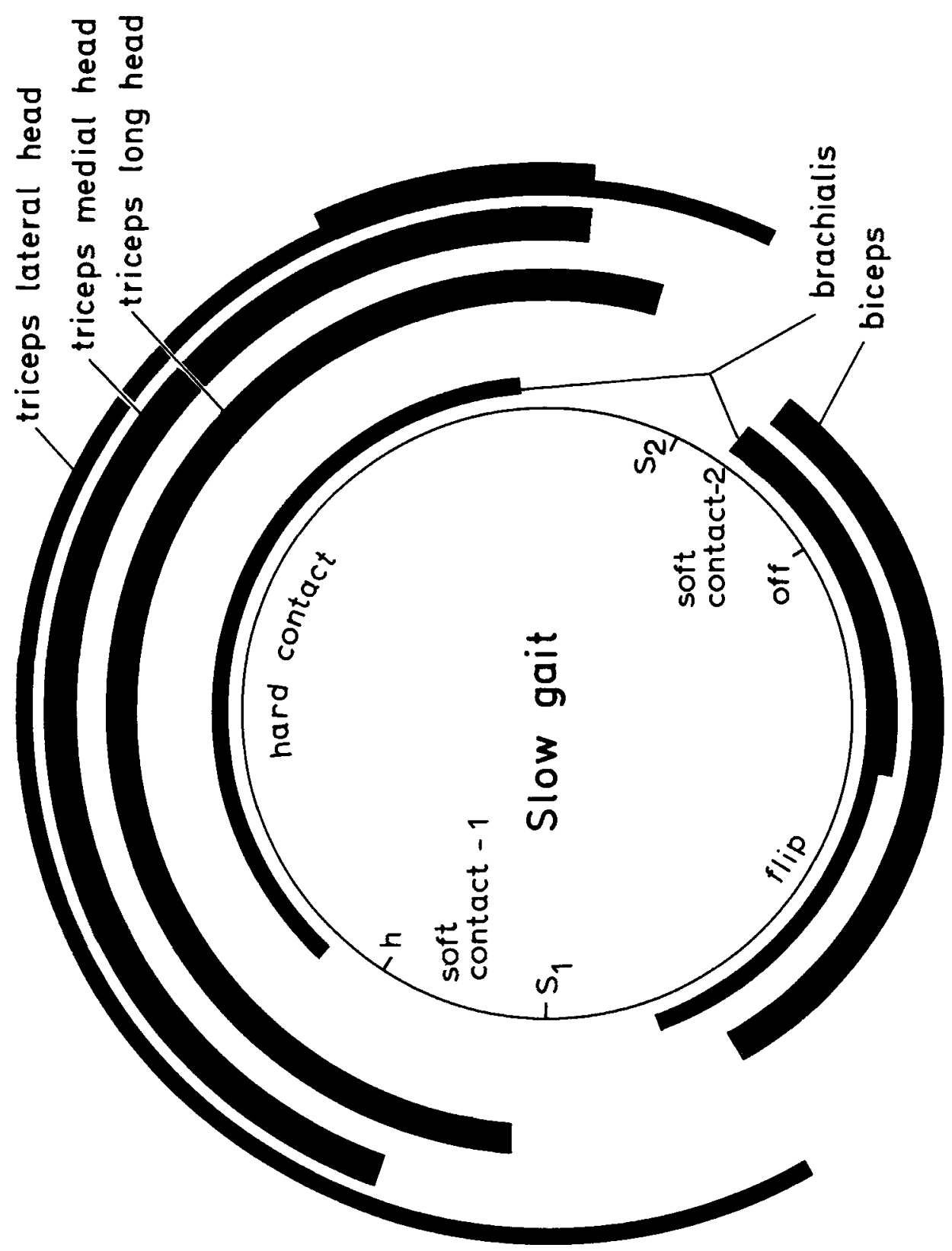

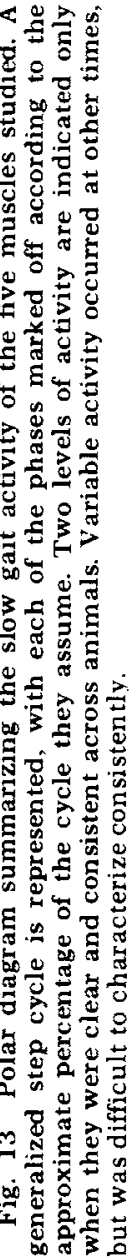




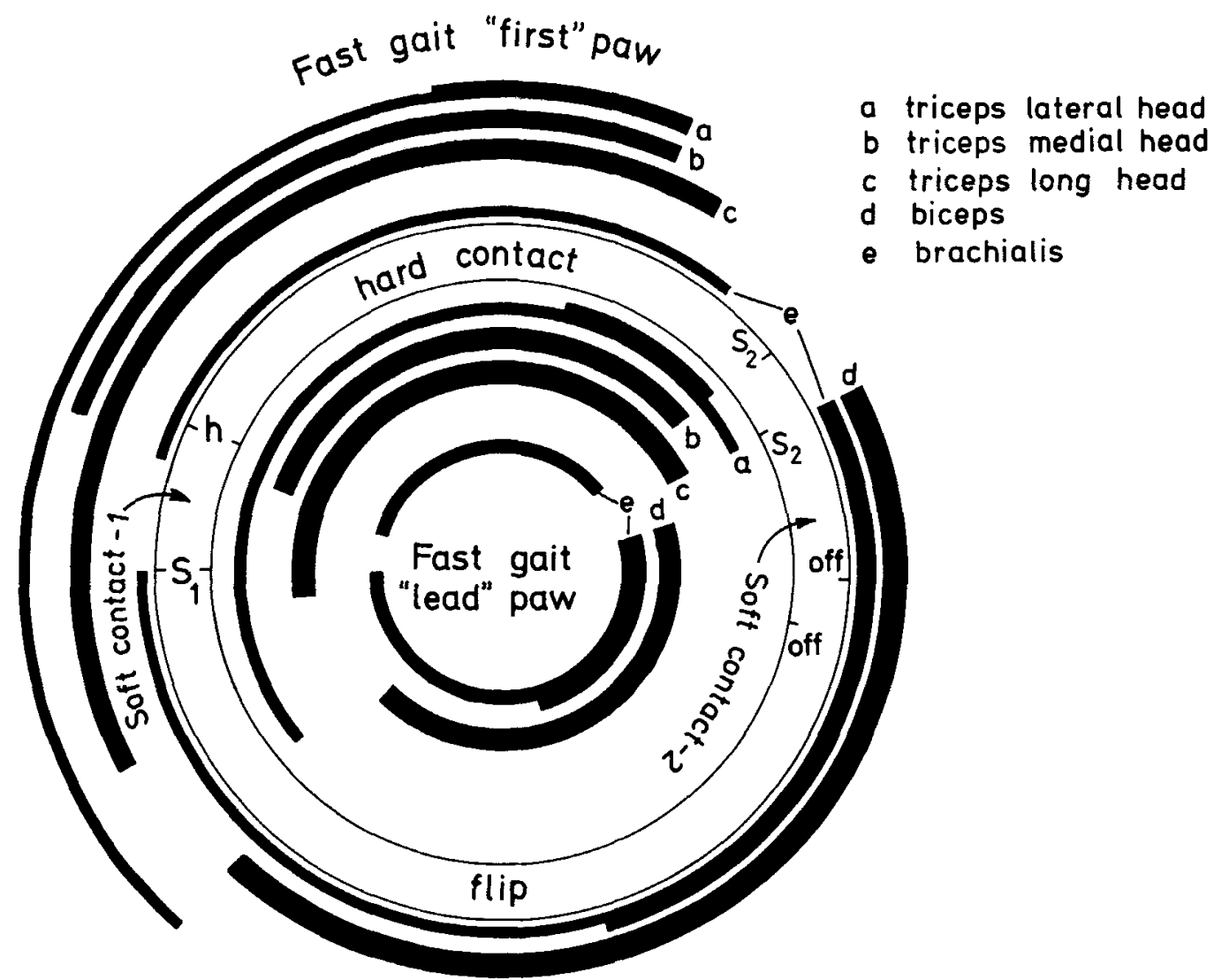

Fig. 14 Polar diagram summarizing fast gait activity of the five muscles studied. Generalized "first" and "lead" paw cycles are drawn on the same diagram to facilitate comparison and to demonstrate the clear asymmetry between them. Conventions as in figure 13.

tion. This suggests that force is exerted, first to place the limb in position and second to support and drive the system during most of this phase. Little extensor activity is observed during the terminal portion of hard contact. The shift of paw from hard to soft contact may well involve inertial forces of the body coupled with movements of the foot and shoulder. All elbow muscles of the "first" paw become silent just before $S_{2}$. In contrast, the lateral and long heads of the triceps fire in the "lead" paw until $S_{2}$; all muscles are silent after $S_{2}$. Thus any propulsive force during soft contact -2 must come from muscles other than the elbow extensors.

The predominant function of providing support is seen especially in the slower gaits, which have a longer hard contact phase during which the bones are held in a relatively fixed position, permitting the transmission of compressive forces to the ground. Further, during walk and trot the extensors fire almost entirely during hard contact (fig. 12). The low-level activity of the lateral head of the triceps and that of the brachialis may provide an additional stabilizing influence to prevent lateral buckling at the elbow joint. As the long head of the triceps is active before contact, it may brace the limb and keep it from buckling during initial contact.

The high-level firing of the lateral head and sustained high-level firing of the long head at the end of the hard contact phase suggest that the forelimb assists in propulsion, as the contralateral forelimb and at least on hindlimb also have hard con- 
tact with the ground near the end of the forelimb's hard contact phase. Consequently, the burst of muscular activity could be propulsive in function. The lateral and long heads take their origin from the scapula and may swing the limb as a whole rather than just the distal portion. The medial head which originates on the shaft of the humerus, in contrast, acts only across a single joint.

The flexors, biceps and brachialis, fire once the limb is being moved anteriorly in the flip. They may function to keep the manus from dragging during this phase. Once the paw is foreward and the manus straightened the biceps ceases firing. The brachialis changes from a high to a low level midway through the flip and maintains this level almost to $S_{1}$.

The rat must retract the limb as the body moves foreward throughout soft contact-1 and hard contact. Retraction is apparently powered by the combined and varying levels of triceps activities as well as those of the shoulder muscles. The surface inclination, texture, speed, weight and position of the animal, as well as the particular gait will influence the motor sequence. It remains to be seen which of these factors accounts for the significant variability in levels of triceps activities from stride to stride and animal to animal. (The interspecimen variation of activity level might, of course, reflect electrode position if the fast and slow fibers were not randomly distributed.)

As the animal switches to faster, more asymmetrical gaits, activities change in relative importance. As one can see by comparing figures 13 and 14 , the flexors fire for a relatively longer period as the flip phase becomes longer and hard contact shorter, especially for the first paw (fig. 9).

Furthermore the nature of the support function changes. In the gallop the hindlimbs accelerate the trunk while the forelimbs are off the ground. The forelimbs must keep the trunk off the ground without too much absorption of kinetic energy, as the latter would reduce efficiency. The long head of the triceps fires sooner in the cycle keeping the limb "under" the trunk; the long head fires before $S_{1}$ even in the lead paw. In the first paw the long head of the triceps lengthens the stride by ex- tending the limb and adding a reaching component to the flip phase. The greater extensor activity may brace the limb so that it can bear the brunt of the impact. The complexity of firing overlap also parallels that reported by Carlsöö and Molbech ('66). Very shortly after the lead paw contacts the ground it must support the body (fig. 8); the hindlimbs are then swinging forward and the first paw will have started the next protraction.

\section{Motor control}

The temporal relationship between the onset of muscle firing and particular limb activities is remarkably constant through a large change in cycle length. The brachialis and medial head of the triceps, for example, consistently commence activity at the same respective point in the step cycle regardless of gait. In contrast, the long head of the triceps shifts its onset both relative to that of other muscles and to limb position (fig. 14), across gaits and across paws even within a single gait. Any speculations on motor control must take into account such constancies and variabilities in the temporal relationships of muscle activities and events of the step cycle. The functional asymmetry of movement and motor activity implies that any central program of motor control must be capable of inducing motion on either side and of giving instructions that may be reversed when an animal changes lead during the run.

The firing of some muscles, such as the biceps, correlates better with one event in the step cycle than another (fig. 11). This may reflect the differential influence of sensory connections from the foot pad, from muscle, or from joint afferents. Such a differential synaptic organization on a population of motor units has been demonstrated in the medial gastrocnemius of the cat (Burke et al., '73). Its presence would have to be demonstrated in the rat before any conclusions could be drawn.

A sharp distinction between flexors and extensors seems spurious in the view of the continuous activity of the brachialis during the "extensor phase" of the step cycle. Similar low-level firing interspersed with occasional high-level bursts is often seen in the biceps during hard contact phase. However, the latter muscle acts more ir- 
regularly, and its activity is difficult to correlate with obvious mechanical events. This diversity of muscle function was long ago discussed by Wilson under the heading "The lore of the antagonists" ('28).

The firing of flexors during hard contact could be explained by the activation of spindle primaries during passive stretch observed by Severin et al. ('67) and discussed by Granit ('70). The lateral head of the triceps also has significant activity when the limb is flexed in the flip phase. The lateral head inserts distally on the ulna; it may be stretched sooner than are the other heads and consequently show an earlier la afferent monosynaptic "reflex" firing. Mendell and Henneman ('71) observed that all $l a$ afferents from a given muscle synapse once and only once on every homonymous motoneuron. The tonic slow twitch muscle units of the hindlimb muscle of the cat have been demonstrated to have a lower threshold for la primary spindle activation than do the more phasic fast units (Burke et al., '73). Thus, low level la afferent activity would primarily affect slow tonic fibers in this population. It is conceivable that the low level activity preceding paw placement reflects the distal insertion of the lateral head. The same argument may apply to the sustained activity of the brachialis during the passive stretch of the hard contact phase. All such speculations need to be tested by further experiments.

\section{ACKNOWLEDGMENTS}

This investigation was supported by $\mathrm{Na}$ tional Science Foundation grant BMS 7101380. We thank Drs. Frits de Vree, C. O. Diefenbach, and Stephen Easter for technical assistance, and Elizabeth Lenneberg for reading the manuscript.

\section{IITERATURE CITED}

Barnett, S. A. 1963 The Rat, a Study in Behavior. Aldine, Chicago.

Burke, R. E., W. Z. Rymer and J. V. Walsh, Jr. 1973 Functional specialization in the motor unit population of cat medial gastrocnemius muscle. In: Control of Posture and Locomotion. R. B. Stein, K. B. Pearson, R. S. Smith and J. B. Redford, eds. Plenum Publ. Corp., New York, pp. 29-44.

Cameron, W. E., D. G. Stuart and G. E. Goslow, Jr. 1973 An analysis of ankle flexion of the cat hindlimb. Amer. Zool., 13: 1343 (abstract). Carlsöö, S., and S. Molbech 1966 The functions of certain two-joint muscles in a closed muscular chain. Acta Morph. Neerl. Scand., 6(4): 377386.

Engberg, I., and A. Lundberg 1969 An electromyographic analysis of muscular activity in the hindlimb of the cat during unrestrained locomotion. Acta Physiol. Scand., 75: 614-630.

Ewer, R. F. 1971 The biology and behaviour of a free-living population of black rats (Rattus rattus). Anim. Behav. Monogr., 4: 125-174.

Gambarian, P. P. 1972 The Running of Mammals [Russian] . Nauka, Leningrad.

Gans, C. 1974 Biomechanics: Approach to Ver tebrate Biology. J. P. Lippincott, Philadelphia.

Goslow, G. E., Jr., R. M. Reinkiney and D. G. Stuart 1973 The cat step cycle: Hindlimb joint angles and muscle lengths during unrestrained locomotion. J. Morph., $141: 1-42$.

Goslow, G. E., Jr., W. E. Cameron and D. G. Stuart $1973 \mathrm{~b}$ Motor units of flexor and extensor muscles of the cat hindlimb. Amer. Zool., 13 . 1343 (abstract).

Granit, R. 1970 The Basis of Motor Control. Academic Press, London.

- 1972 Mechanisms Regulating the Discharge of Motoneurons. Charles $\mathrm{C}$ Thomas, Springfield.

Greene, E. 1935 Anatomy of the rat. Trans. Amer. Phil. Soc., Vol. 27.

Hildebrand, $M .1962$ Walking, running, and jumping. Amer. Zool., 2: 151-155.

1965 Symmetrical gaits of horses. Science, 150: 701-708.

1966 Analysis of the symmetrical gaits of tetrapods. Fol. Biotheor., 6: 9-22.

1974 Analysis of Vertebrate Structure. John Wiley and Sons, New York.

Howell, A. B. 1944 Speed in Animals. University of Chicago Press, Repr. (1965) Hafner Publ. Co., New York.

Jenkins, F. A,, Jr. 1971 Limb posture and locomotion in the Virginia opossum (Didelphis marsupialis) and in other non-cursorial mammals. J. Zool., London, 165: 303-315.

- 1973 The functional anatomy and evolution of the mammalian humero-ulnar articu lation. Amer. J. Anat., 137: 281-297.

1974 Primate Locomotion. Academic Press, New York.

Mendell, L. M, and E. Henneman 1971 Terminals of single la fibers: location, density and distribution within a pool of 300 homonymous motoneurons. J. Neurophysiol., 34 : 171-187.

Oxnard, C. E. 1973 Some locomotor adaptations among lower primates: Implications of primate evolution. Symp. Zool. Soc. London, 33: 255299.

Severin, F. V., G. N. Orlovskij and M. L. Shik 1967 Work of the muscle receptors during controlled movement. Biofizika, 12: 502-511.

Sperry, R. W. 1940 The functional results of muscle transposition in the hindlimb of the rat. J. Comp. Neur., 73: 379-404.

- 1941 The effect of crossing nerves to antagonistic muscles in the hindlimb of the rat. J. Comp. Neur., 75 : 1-19.

1942 Transplantation of motor nerves and muscles in the for elimb of the rat. J. Comp. Neur., $76 \cdot 283-321$.

Sukhanov, V. B. 1968 General System of Sym- 
metrical Locomotion of Terrestrial Vertebrates and Some Features of Movement of Lower Tetrapods. Nauka Publishers, Leningrad; English translation (1974) Amerind Publ. Co., New Delhi.

Taylor, M. E. 1970 Locomotion in some East African viverrids. J. Mamm., 51: 42-51.

Tricker, R. A. R., and B. J. K. Tricker 1967 The Science of Movement. American Elsevier, New York.
Vree, F. de, and C. Gans 1975 Mastication in pygmy goats, Capra hircus. Ann. Soc, Zool. Belge (accepted).

Wilson, S. A. K. 1929 Modern Problems in Neurology. William Wood, New York.

Zimmerman, E. A., W. W. Chambers and C. N. Liu 1964 An experimental study of the anatomical organization of the cortico-bulbar system in the albino rat. J. Comp. Neur., 123: 301324. 